\title{
Algunas reflexiones sobre fragmentación y sus retos para la investigación
}

\section{Some reflections about fragmentation and their challenges for research}

\author{
Andrés Quintero-Ángel ${ }^{1,2}$, Daniel Osorio-Domínguez ${ }^{3}$, Leonor Valenzuela ${ }^{4}$
}

\begin{abstract}
Resumen
La fragmentación y pérdida de hábitat son unas de las principales amenazas para la biodiversidad; se alteran procesos ecológicos importantes para la regulación de los ecosistemas que eventualmente pueden estar asociados con procesos de extinción de especies. La respuesta de las especies a estos fenómenos se da a diferentes escalas espaciotemporales, convirtiendo el estudio de la fragmentación y su efecto sobre las especies en un reto y una necesidad urgente para la conservación de las mismas. En este sentido el presente artículo examina el efecto de la fragmentación sobre las interacciones ecológicas, niveles organizacionales, al tiempo que se evalúa de manera general, el estado de la investigación sobre el tema en Latinoamérica.
\end{abstract}

Palabras clave: Fragmentación del hábitat; Interacciones ecológicas; Latinoamérica.

\begin{abstract}
Fragmentation and habitat loss are some of the major threats to biodiversity, altered ecological processes important for the regulation of ecosystems that may eventually be associated to processes of species extinction. The response of the species to these phenomena occurs at different scales of space-time, making the study of fragmentation and its effect on species in a challenge and an urgent need for the conservation. In this sense this article examines the fragmentation impacts on the ecological interactions, organizational levels and at the same time, we evaluate in a general way the state of the research on the topic in Latin America.
\end{abstract}

Keywords: Habitat fragmentation; Ecological interactions; Latinoamerica.

\section{Introducción}

Desde finales del siglo XIX, el aumento significativo de las actividades humanas ha traído consecuencias sobre los procesos ecológicos en sus diferentes estados organizacionales. Uno de los principales resultados de estas actividades es la fragmentación del hábitat, siendo ésta una de las principales amenazas a la biodiversidad, sobre todo en regiones con altos índices de diversidad biológica ya sea considerada como riqueza de especies, diversidad genética o funcional (Kattan 2002, Hoekstra et al. 2005, Kereiva y Marvier 2011). Farigh (2003) ha definido la fragmentación como un proceso donde una gran área de hábitat es transformada en muchas áreas de menor tamaño, incrementando el aislamiento entre los remanentes resultando en la pérdida de hábitat, las alteraciones de diferentes procesos ecológicos que actúan a diferentes escalas y eventuales procesos de extinción (Turner 1996, Kattan y Murcia 2003).

Desde esta perspectiva, la extinción de especies se interpreta entonces como el resultado de fenómenos que ocurren a diferentes escalas tanto temporales como espaciales. Diamond (1972) señala la existencia de un proceso de «relajación» que ocurre en

Pontificia Universidad Javeriana, Facultad de Estudios Ambientales y Rurales, Bogotá, Colombia. e-mail: aquinteroa@gmail.com SENSE. Social and Environmental Sense, Cali, Colombia.

Universidad de Chile, Departamento de Ciencias Ecológicas, Facultad de Ciencias, Santiago, Chile.

Pontificia Universidad Católica de Chile, Departamento de Ecología, Facultad de Ciencias Biológicas, Instituto de Ecología y Biodiversidad (IEB), Santiago, Chile.

Fecha recepción: Enero 12, 2012

Fecha aprobación: Abril 10, 2012

(c) Rev. Biodivers. Neotrop. 2012; 2 (1): 15-20 
la escala temporal. Este proceso conocido como deuda de extinción (Tilman et al. 2004), es resultado de una respuesta retardada de las especies a los cambios en calidad de hábitat y en algunos procesos ecológicos y ecosistémicos resultantes de la fragmentación. La fragmentación como proceso afecta de manera diferencial a las especies según las características biológicas de las mismas; por ejemplo, los grandes depredadores con grandes rangos vitales y bajas tasas reproductivas tienen mayores probabilidades de extinción (Kuussaari 2009). A escala espacial la fragmentación actúa a dos niveles: i) a escala regional, en donde la reducción de la cobertura vegetal reduce la diversidad y disponibilidad de hábitats y ii) a escala local, donde la fragmentación deja las poblaciones aisladas en remanentes de bosque por lo general pequeños, aumentando el riesgo de extinción ya sea por factores estocásticos o determinísticos (Kattan 2002). En este sentido, la estocasticidad demográfica, la pérdida de variabilidad genética por endogamia y la limitada capacidad de migración de las poblaciones en respuesta a las perturbaciones son factores que sumados a la degradación del hábitat interactúa aditiva y sinérgicamente con el efecto de área trayendo múltiples efectos a nivel poblacional, comunitario y ecosistémico causando la pérdida de especies (Collinge 2009).

Por consiguiente, el fenómeno de la fragmentación por pérdida de hábitat se ha convertido en uno de los principales temas de investigación abordado por los biólogos de la conservación (Fazey et al. 2005, Grez y Galetto 2011), porque sus efectos usualmente negativos para la biodiversidad, representan una gran amenaza para un buen número de grupos taxonómicos, sobre todo los especialistas de hábitat (Didham et al. 1996, Gibbons et al. 2000, Hobbs y Yates 2003). Sin embargo, la mayor parte de las investigaciones que miden estos efectos de la fragmentación se han enfocado a escala de sitio/fragmento y no a escala de paisaje, donde sus efectos son más evidentes; además se confunde la fragmentación per se con la pérdida de hábitat, los cuales según Farigh (2003), son dos procesos diferentes, donde es esta pérdida de hábitat, el proceso con efectos negativos sobre la biodiversidad. Asimismo, los efectos e importancia de la matriz, los modelos metapoblaciones y los experimentos naturales manipulados han sido escasamente abordados. Muchos de los estudios en fragmentación se han enfocado en unas pocas especies o en grupos taxonómicos particulares (Lindenmayer y Fisher 2006).

\section{¿Qué sabemos sobre la fragmentación y sus efectos en los niveles organizacionales?}

Factores como el área, el aislamiento y la estructura vegetal de los fragmentos son de suma importancia porque estas características se encuentran ligadas a la historia natural de cada una de las especies (Zimmerman y Bierregaard 1986, Cushman 2006). Puesto que los cambios en las condiciones ambientales generados por la fragmentación por pérdida de hábitat, actúan sobre cada especie de manera independiente, cada una responde a las condiciones ecológicas de manera particular (Marsh y Pearman 1997, Pineda y Halffter 2004). Por esta razón, es necesario entender las interacciones intraespecíficas e interespecíficas, así como los procesos ecológicos que establecen las especies con el ambiente.

Aquí cobra gran importancia la teoría de nicho (Hutchinson 1957, MacArthur 1968), que hace referencia al espacio multidimensional inherente a cada especie para permitir una óptima adecuación biológica (Leibold 2003). Esto determina que las interacciones de las especies con el ambiente difieran de manera significativa a diferentes escalas (Pineda y Halffter 2004). Por esto es importante evaluar las interacciones entre múltiples atributos ambientales, a través de un rango de escalas para un paisaje completo que contenga docenas o cientos de poblaciones locales (Pineda y Halffter 2004). Solo analizando la relevancia del hábitat para cada una de las especies puede ser posible hacer verdaderas inferencias acerca de los impactos de la pérdida de hábitat y la fragmentación en las poblaciones (McGarigal y Cushman 2002).

La pérdida de hábitat y la fragmentación tienen efectos con respecto a las relaciones entre poblaciones de la misma especie, porque se generan cambios en la biología, comportamiento, alteraciones del rango vital, la fenología o interrupción en los patrones de distribución y dispersión entre otros factores de cada especie en particular (Ims et al. 1993, Fisher y Lindenmayer 2007, Zitske et al. 2011). Por otra par- 
te, la fragmentación puede alterar las relaciones entre conspecíficos, convirtiendo las características endógenas de las especies en posibles factores de amenaza para la supervivencia de las poblaciones en el tiempo en hábitats fragmentados (Fisher y Lindenmayer 2007). Sin embargo, sistemas fragmentados con remanentes lo suficientemente grandes para mantener poblaciones locales viables, teniendo en cuenta la dinámica de las metapoblaciones, aumentan la probabilidad de sobrevivencia a eventos tanto determinísticos como estocásticos tales como depresión por endogamia, epidemias, perturbaciones naturales y antrópicas (Hanski y Gaggiotti 2004). Asimismo, para especies pequeñas y de comportamientos agregados como los anfibios, la fragmentación del hábitat no necesariamente representa un riesgo para su viabilidad. Por lo tanto, a nivel meta-comunitario, en sistemas fragmentados con alta heterogeneidad ambiental y baja conectividad, la diversidad beta aumenta, incrementando de esta manera la diversidad gamma (Forbes y Chase 2002, Economo y Keitt 2008).

Finalmente, uno de los niveles organizacionales en ecología de ambientes fragmentados que menos se ha abordado a pesar de su vital importancia, es el nivel ecosistémico. Teóricamente se espera que debido a los cambios abióticos y la disminución de la diversidad por efecto de la fragmentación por pérdida de hábitat, se vean afectadas diferentes funciones relativas a este nivel como la descomposición, el reciclaje de nutrientes o el control biológico (Cardinale et al. 2012). Por lo tanto, es necesario empezar a entender y cuantificar el efecto de la fragmentación sobre estas funciones que no solo son valiosas para el buen funcionamiento de dichos fragmentos, sino que terminan trascendiendo en el bienestar humano gracias a su indisoluble relación con los bienes y servicios ecosistémicos (Costanza et al. 1997).

\section{¿Qué sabemos sobre la fragmentación y sus efectos en las interacciones ecológicas?}

Otros procesos ecológicos afectados por la fragmentación del hábitat son la competencia, la depredación y el mutualismo (Fisher y Lindenmayer 2007). Estas alteraciones pueden desencadenar cascadas tróficas, si se ven involucradas especies claves (keystone species), que mantienen o cumplen funciones vitales del ecosistema como es el caso de los depredadores tope (Soulé y Terborgh 1999, Estes et al. 2011).

Interacciones mutualistas planta-animal, como la polinización y la dispersión de semillas son cruciales por su papel fundamental en los procesos de regeneración, establecimiento de comunidades vegetales y la subsistencia de la biodiversidad (Wang y Smith 2002, Beckage y Clark 2005). Considerando que a través de la movilidad de semillas por parte de frugívoros y la dispersión de gametos a través de la polinización se asegura la dispersión y variabilidad de las especies vegetales en un área determinada, se puede mejorar la conectividad funcional a través del paisaje, contribuyendo incluso con la recuperación del mismo (Bodin et al. 2006, García et al. 2010).

Interacciones como la herbivoría y la insectívora también se ven afectadas de diferentes maneras. Tscharntke y Brandl (2004) sugieren que existe mucha contingencia en estas relaciones, algunas especies responden de forma positiva y otras negativamente. En los bosques templados de Chile los insectos son los herbívoros más importantes y su abundancia varía en forma compleja entre los fragmentos y el bosque continuo (Jaña-Prado y Grez 2004, De la Vega y Grez 2008). En los fragmentos la abundancia de insectos herbívoros es menor, expresándose así una menor defoliación, pero el efecto de dicho fenómeno sobre plántulas y árboles adultos es diferencial. En bosques tropicales el patrón es el mismo, sin embargo, a medida que pasa el tiempo de fragmentación, los niveles de herbivoría en los fragmentos se nivelan con los niveles del bosque continuo (Benítez-Malvido et al. 1999). Por otra, parte la insectívora en bosques templados y tropicales es menor en los fragmentos de pequeños, debido a la disminución significativa en la riqueza y abundancia de aves insectívoras (Willson et al. 1994, Sekercioglu et al. 2002). Sin embargo, este no es el patrón que se observa en algunos bosques centrales de Chile, donde la abundancia de aves insectívoras en los fragmentos es mayor que en los fragmentos de mayor tamaño (Vergara y Simonetti 2004, González-Gómez et al. 2006).

Sin embargo, hay que recordar que estas interacciones cambian según las especies y la escala, por 
lo que los efectos de la fragmentación sobre estas interacciones podría estar relacionadas con cambios en la configuración espacial del hábitat, en lugar de la pérdida de la misma (Farwig et al. 2009, Herrera, et al. 2011). Además dependiendo de la geografía será el resultado de la variación espacial en la dinámica ecológica entre planta-animal (de la Peña et al. 2011).

\section{¿Qué sabemos del tema en Latinoamérica?}

Aunque la producción literaria en temas sobre fragmentación se ha incrementado en los últimos años, a nivel latinoamericano no existen revisiones sobre el estado del arte de este tema (Grez y Galleto 2011). En este sentido Brasil y México son los países con mayor cantidad de estudios relacionados con este tema donde se han publicado hasta la fecha, según la base de datos ISI Web of Science, 217 y 130 artículos respectivamente (Figura 1). Para un país como Colombia donde es reconocido como uno de los países con mayor diversidad en especies biológi- cas, su producción científica en el tema de la fragmentación ocupa la séptima posición en Latinoamérica (Grez y Galleto 2011) con tan solo veinte artículos (Figura 1). No menos preocupante, es que para los estudios existentes hay regiones en Latinoamérica que se encuentran subrepresentadas.

Un buen ejemplo de la ausencia de investigaciones en el tema de la fragmentación lo constituye la cordillera de los Andes, para la cual a pesar de haber estado sometida a procesos de modificación antrópica incluida la fragmentación por cuenta de la actividad humana desde tiempos precolombinos (Eter y VanWyngaarden, 2000), el número de publicaciones que hacen referencia al tema encontradas en la base de datos ISI Web of Science son pocas $(\mathrm{N}=25)$, la mayoría realizadas en Colombia (13 publicaciones). Esto sin mencionar la inexistencia de literatura sobre fragmentación que haga referencia a zonas como el Chocó Biogeográfico, lo cual es aun más relevante si se tiene en cuenta que esta región es reconocida como importante centro de biodiversidad y endemismo gracias a su particular historia biogeográfica (Myers et al. 2000).

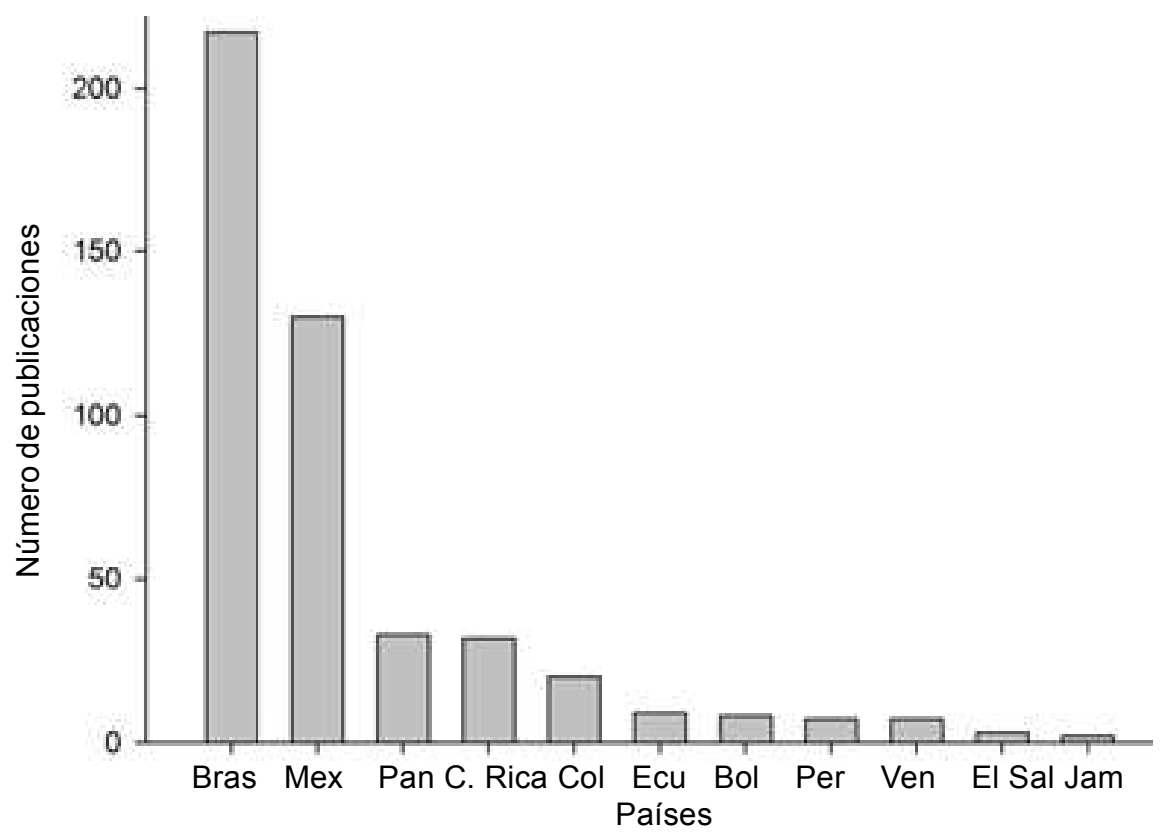

Figura 1. Número absoluto de trabajos publicados sobre fragmentación en los países del neotrópico según la base de datos ISI Web of Science.

Bras: Brasil, Mex: México, Pan: Panamá, C. Rica: Costa Rica, Col: Colombia, Ecu: Ecuador, Bol: Bolivia, Per: Perú, Ven: Venezuela, El Sal: El Salvador, Jam: Jamaica. 


\section{Conclusiones}

Actualmente, las actividades humanas han modificado considerablemente los paisajes naturales a través de diferentes procesos dentro de los cuales se incluye la fragmentación, alterando de esta manera la distribución y abundancia de plantas y animales (Lindenmayer y Fisher 2006). Estos cambios en las especies dependen de la autoecología de cada una de estas, el lugar (hábitat), la escala espacial y el tiempo e historia de los procesos de fragmentación (Power, et al. 1996, de la Peña, et al. 2011). Bajo este escenario, sin embargo, persisten poblaciones viables, dinámicas ecológicas y procesos ecosistémicos. Es por esta razón que desde la perspectiva de la biología de la conservación y bajo los escenarios actuales se debe apuntar no solo a conservar hábitats poco perturbados (cada vez más escasos) sino que se deben incluir paisajes fragmentados en donde en conjunto todos los fragmentos, se pueden complementar para mantener una mayor diversidad de especies en las diferentes regiones.

Como los cambios en las variables bióticas y abióticas en cada uno de los fragmentos actúan de manera diferente, y por lo tanto repercuten de manera diferencial en la configuración poblacional, comunitaria y las funciones ecosistémicas, es necesario entender cuáles son los procesos y factores que determinan dicha persistencia de las especies y sus dinámicas en estos fragmentos, para poder desarrollar políticas de conservación eficientes. Esto solo se puede lograr aumentando las aproximaciones metodológicas, en especial aquellas experimentales y estudiando procesos poco abordados en este tema como el efecto de la pérdida de biodiversidad por efecto de la fragmentación en las funciones ecosistémicas.

\section{Agradecimientos}

Los autores agradecemos a los dos revisores anónimos por sus valiosos comentarios.

\section{Literatura citada}

Beckage B, Clark JS. 2005. Does predation contribute to tree diversity? Oecologia. 143: 458-69.

Bemitez-Malvido J, García-Guzmán G, Kossman-Ferraz ID.
1999. Leaf-fungal incidence and herbivory on tree seedlings in tropical rainforest fragments: an experimental study. Biol Conserv. 91: 143-50.

Bodin Ö, Tengö M, Lundberg AN J, Elmqvist T. 2006. The value of small size: loss of forest patches and ecological thresholds in southern Madagascar. Ecol Applic. 16: 44051.

Cardinale BJ, Duffy JE, Gonzáles A, Hooper DU, Perrings C, Venail $\mathrm{P}$, et al. 2012. Biodiversity loss and its impact on humanity. Nature. 486: 59-67.

Collinge SK. 2009. Ecology of fragmented landscape. Baltimore: The Johns Hopkins University Press.

Cordeiro NJ, Ndangalasi HJ, McEntee JP, Howe HF. 2009. Disperser limitation and recruitment of an endemic African tree in a fragmented landscape. Ecology. 90: 1030-41.

Costanza R, d'Arge R, de Groot R, Farber S, Grasso M, Hannon $\mathrm{B}$, et al. 1997. The Value of the world's ecosystem services and natural capital. Nature. 387: 253-60.

Cushman SA. 2006. Effects of habitat loss and fragmentation on amphibians: A review and prospectus. Biol Conservat. 128: 231-40.

De la Peña E, D’hondt B, Bonte D. 2011. Landscape structure, dispersal and the evolution of antagonistic plant-herbivore interactions. Ecography. 34: 480-7.

De la Vega J, Grez AA. 2008. Composición, riqueza de especies y abundancia de insectos defoliadores de actividad nocturna asociados a Aristotelia chilensis (maqui) en el bosque maulino fragmentado. Rev Chilena Hist Nat. 81: 221-38.

Diamond JM. 1972. Biogeographic kinetics: estimation of relaxation times for avifaunas of southwest pacific islands. Proc Nat Acad Sci USA. 69: 3199-203.

Didham RK, Ghazoul J, Stork NE, Davis AJ. 1996. Insects in fragmented forest: a functional approach. Trends Ecol Evol. 11: 255-60.

Economo EP, Keitt TH. 2008. Species diversity in neutral metacomunities: a network approach. Ecol Letters. 11: 52-62.

Etter A, van-Wyngaarden W. 2000. Patterns of landscape transformation in Colombia, UIT emphasis in the Andean Region. Ambio. 29: 432-9.

Estes JA, Terborgh J, Brashares JS, Power ME, Berger J, Bond WJ, et al. 2003. Effects of habitat fragmentation on biodiversity. Ann Rev Ecol Syst. 34: 487-515.

Farwig, N, Bailey D, Bochud E, Herrmann JD, Kindler E, Reusser N, et al. 2009. Isolation from forest reduces pollination, seed predation and insect scavenging in Swiss farmland. Landscape Ecol. 24: 919-27.

Fazey I, Fischer J, Lindenmayer DB. 2005. What do conservation biologists publish? Biol Conserv. 124: 6373.

Fisher J, Lindenmayer DB. 2007. Landscape modification and habitat fragmentation: a synthesis. Global Ecol Biogeog. 16: $265-80$

Forbes AE, Chase JM. 2002. The role of habitat connectivity and landscape geometry in experimental zooplankton metacomunities. Oikos. 96: 433-40. 
García D, Zamora R, Amico GC. 2010. Birds as suppliers of seed dispersal in temperate ecosystems: conservation guidelines from real-world landscapes. Conserv Biol. 4: 1070-9.

Gibbons JW, Scott DE, Ryan TJ, Buhlmann KA, Tuberville TD, Metts BS, et al. 2000. The global decline of reptiles, déjá vu amphibians. Bioscience. 50: 653-66.

González-Gómez PL, Estades CF, Simonetti JA. 2006. Strengthened insectivory in a temperate fragmented forest. Oecologia. 148: 137-43.

Hanski I, Gaggiotti OE. 2004. Ecology, genteics, and evolution of metapopulations. San Diego: Elseviere Academic Press.

Hutchinson GE. 1957. Concluding remarks. Cold Springs Harbor Symposia on Quantitative Biology, 22: 415-27.

Grez A, Galetto L. 2011. Fragmentación del paisaje en América Latina: ¿en qué estamos? 63-78 En: Simonetti, J, Dirzo $\mathrm{R}$ (eds). Conservación biológica: perspectivas desde América Latina. Santiago: Editorial Universitaria.

Herrera JM, García D. 2009. The role of remnant trees in seed dispersal through the matrix: being alone is not always so sad. Conservat Biol. 142: 149-58.

Hobbs RJ, Yates CJ. 2003. Impacts of ecosystem fragmentation on plant populations: generalizing the idiosyncratic. Australian J Bot. 51: 471-88.

Hoekstra JM, Boucher TM, Ricketts TH, Roberts C. 2005. Confronting a biome crisis: global disparities of habitat loss and protection. Ecol Letters. 8: 23-9.

Ims RA, Rolstad J, Wegge P. 1993. Predicting space use responses to habitat fragmentation: can voles Microtus oeconomus serve as an experimental model system (EMS) for Capercaillie Grouse Tetrao urogallus in boreal forest? Biol Conservat. 63: 261-68.

Jaña-Prado R, Grez AA. 2004. Insectos herbívoros en el bosque maulino: un ecosistema fragmentado. Rev Chilena Entomol. 30: 27-43.

Kattan G H. 2002. Fragmentacion: patrones y mecanismos de extinción de especies. En: Guariguata MR, Kattan GH (eds). Ecología y conservación de bosques tropicales. San José: Ediciones LUR.

Kattan GH, Murcia C. 2003. A review and synthesis of conceptual frameworks for the study of forest fragmentation. In: Bradshaw GA, Marquet PA (eds). Ecological estudies. Vol 162. Berlin: Springer-Verlag.

Kareiva PM, Marvier M. 2011. Conservation science: Balancing the needs of people and nature. Greenwood Village: Roberts and Co.

Kuussaari M, Bommarco R, Heikkinen RK, Helm A, Krauss J, Lindborg R, et al. 2009. Extinction debt: a challenge for biodiversity conservation. Trends in Ecol Evol. 24: 56471.

Leibold M. (2003). Ecological niches: linking classical and contemporary approaches. Chicago: University of Chicago Press.
Lindenmayer DB, Fisher J. 2006. Habitat fragmentation and landscape change: an ecological and conservation synthesis. Washington, DC: Island Press.

MacArthur R. 1968. The theory of the niche. En: RC Lewontin (ed). Population biology and evolution. Syracuse: Syracuse University Press. Pp. 159-76.

Marsh DM, Pearman PB. 1997. Effects of habitat fragmentation on the abundance of two species of leptodactylid frogs in Andean montane forest. Conservat Biol. 11: 1323-8.

McGarigal K, Cushman SA. 2002. Comparative evaluation of experimental approaches to the study of habitat fragmentation effects. Ecol Applicat. 12: 335-45.

Myers N, Mittermeier RA, Mittermeier CG, da Fonseca GAB, Kent J. 2000. Biodiversity hotspots for conservation. Nature. 403: 853-8.

Pineda E, Halffter G. 2004. Species diversity and habitat fragmentation: frogs in a tropical montane landscape in Mexico. Biol Conservat. 117: 499-508.

Power ME, Tilman D, Estes JA, Menge BA, Bond WJ, Mills LS, et al. 1996. Challenges in the quest for keystones. BioScience. 46: 609-20.

Sekercioglu CH, Ehrlich PR, Daily GC, Aygen D, Goehring D, Figeroa-Sandí R. 2002. Disappearance of insectivorous birds from tropical forest fragments. Proc Nat Acad Sci USA. 99: 263-7.

Soulé ME, Terborgh, J. 1999. Protecting nature at regional and continental scales: A conservation biology program for the new millennium. BioScience. 49: 809-17.

Tilman D, May RM, Lehman CL, Nowak MA. 1994. Habitat destruction and the extinction debt. Nature. 371: 65-6.

Tscharntke T, Brandl R. 2004. Plant-insect interactions in fragmented landscapes. Ann Rev Ecol Systemat. 49: 405-30.

Tylianakis JM, Didham RK, Bascompte J, Wardle DA. 2008. Global change and species interactions in terrestrial ecosystems. Ecol Letters. 12: 1351-63.

Turner IM. 1996. Species loss in fragments of tropical rain forest: A review of the evidence. J Appl Ecol. 33: 200-09.

Vergara P, Simonetti JA. 2004. Avian responses to fragmentation of the Maulino Forest in central Chile. Oryx. 4: 1-6.

Wang, BC, Smith TB. 2002. Closing the seed dispersal loop. Trends Ecol Evolut. 17: 379-86.

Willson MF, De Santo TL, Sabag C, Armesto JJ. 1994. Avian communities of fragmented south-temperate rainforests in Chile. Conservat Biol. 8: 508-17

Zimmerman BL, Bierregaard RO. 1986. Relevance of the equilibrium theory of island biogeography and speciesarea relations to conservation with a case from Amazonia. J Biogeograp. 13: 133-43.

Zitske BP, Betts MG, Diamond AW. 2011. Negative effects of habitat loss on survival of migrant warblers in a Forest Mosaic. Conservat Biol. 5: 993-1001. 